\title{
Staining with two observational methods for the diagnosis of tuberculous meningitis
}

\author{
YUELI ZOU* , HUI BU* , LI GUO, YAJUAN LIU, JUNYING HE and XUEDAN FENG \\ Department of Neurology, The Second Hospital of Hebei Medical University, Shijiazhuang, Hebei 050000, P.R. China
}

Received February 17, 2015; Accepted March 17, 2016

DOI: $10.3892 /$ etm.2016.3859

\begin{abstract}
Ziehl-Neelsen (Z-N) staining of cerebrospinal fluid (CSF) for acid-fast bacilli (AFB) is the cornerstone of the laboratory diagnosis of tuberculous meningitis (TBM). However, the sensitivity of conventional Z-N staining for the detection of AFB in CSF specimens is suboptimal. The present study aimed to compare the practicality of modified Z-N staining with light microscopy and fluorescence microscopy in the same smear without auramine O. A total of 155 patients with 223 CSF specimens were enrolled and grouped according to the uniform case definition. The smears of each CSF specimen were subjected to modified Z-N staining and then observed using a light microscope under transmitted light and under fluorescence with a green-excitation wavelength in the same microscopic field. The results for different groups, inspection times, and prior to and following treatment were compared. Results indicated that the fuchsin-stained AFB were visible as bright orange-red fluorescing rods under fluorescence, or as red, lightly curved rods under transmitted light. The sensitivity of fluorescence microscopy was $96.2 \%$ while that of light microscopy was $84.6 \%$. The positive rate of fluorescence microscopy was $79.2 \%$ prior to treatment compared with $61.7 \%$ post-treatment. In the same microscopic field, a greater number of AFB were observed using fluorescence compared with transmitted light, and AFB that were not visible under transmitted light were clearly observed under fluorescence. Furthermore, transmitted light and fluorescence could be interchanged directly when equivocal smears were encountered. The combination of modified Z-N staining and fluorescence microscopy without auramine $\mathrm{O}$ is sensitive and convenient for the diagnosis of TBM.
\end{abstract}

Correspondence to: Dr Junying He, Department of Neurology, The Second Hospital of Hebei Medical University, 215 Peace West Road, Shijiazhuang, Hebei 050000, P.R. China

E-mail: guolicn@126.com

*Contributed equally

Key words: cerebrospinal fluid, fluorescence microscopy, light microscopy, Ziehl-Neelsen staining

\section{Introduction}

Tuberculous meningitis (TBM) is the most lethal form of tuberculosis. A higher proportion of sufferers succumb to mortality as a result of TBM compared with any other form of tuberculosis (1). Rapid diagnosis and early treatment should enable the mortality to be reduced. The ideal tuberculosis screening test should be cost-effective, robust and easy to perform, require little infrastructure or reagents and provide results within a short span of time (2). Of the current diagnostic methods, smear microscopy remains the most convenient gold standard for detecting acid-fast bacilli (AFB), especially in developing countries (3). An international TBM workshop has reported a definite diagnosis criteria for TBM, which is the detection of AFB in the cerebrospinal fluid (CSF) (4). However, the sensitivity of conventional Ziehl-Neelsen (Z-N) staining for CSF specimens is rarely $>60 \%$ (5-10). Modified Z-N staining is advocated as a good technique with high sensitivity and specificity (6). However, a mycobacterium is thin with an approximate length of $1-4 \mu \mathrm{m}$, and the density of mycobacteria in the CSF is low (11). Typically, $\geq 5 \mathrm{~min}$ is required for the reading of a negative smear with light microscopy (12), and further time is required to examine all the visual fields, which increases the workload of the microscopist. Therefore, rapid and simple diagnosis methods are urgently required for the diagnosis of TBM.

In the present study, two observational methods were employed for modified Z-N staining in the same smear. The smears were firstly observed under transmitted light, and then observed by fluorescence directly, without auramine $\mathrm{O}$ or auramine-rhodamine. The aim of the present study was to compare the practicality of the two observational methods and provide a more effective method for the diagnosis of TBM.

\section{Materials and methods}

Subjects. Ethical approval for the present study was granted by the Research Ethics Committees of the Second Hospital of Hebei Medical University (Shijiazhuang, China), and written informed consent was obtained from all patients or their direct relatives. A total of 155 patients from the Second Hospital of Hebei Medical University were enrolled in the study, and 223 CSF specimens were collected from them between March 2011 and March 2013. Among the enrolled patients, 99 patients with 167 specimens were clinically diagnosed 
with TBM according to the uniform case definition for use in clinical research (4). A definitive diagnosis of TBM was made if there was evidence of AFB in the CSF smear, culture or on histological specimens from the brain or spinal cord. According to the point-scoring system reported by a previous study (4), a probable diagnosis was established if the total score was $>10$ points and no imaging data was available for patients, or $>12$ points if imaging was used. A possible diagnosis was made on the basis of scores between 6 and 9 points without imaging or between 6 and 11 points with imaging. Patients were excluded if their diagnostic score was $<6$. The patients were classified as definite, probable and possible TBM and 56 patients with 56 samples in which CSF was found to be the pathogen served as negative controls. Meningeal carcinomatosis was confirmed according to the detection of malignant cells from the CSF. Cryptococcal meningitis was confirmed according to the detection of Cryptococcus neoformans or culture isolation of Cryptococcus neoformans from CSF. Viral encephalitis was confirmed according to etiological diagnosis (CSF had a positive antigen screening). Leukemia with central nervous system involvement was confirmed by the detection of leukemia cells in CSF. Purulent meningitis was identified through the culturing of CSF and neurocysticercosis was diagnosed based upon clinical characteristics, imaging findings and histological changes.

In order to analyze the changes of the positive rate of modified Z-N staining over time, all patients were classified into two stages according to the duration of symptoms prior to admission to the Second Hospital of Hebei Medical University (stage 1, <1 month prior to admission to the hospital; stage 2, $\geq 1$ month prior to admission to the hospital).

Modified Z-N staining and GeneXpert test. Standard procedures were performed upon each of the CSF smears: A poly-l-lysine-coated slide was used to collect the cells in $0.5 \mathrm{ml} \mathrm{CSF}$ specimen by centrifugation at $150 \mathrm{x} \mathrm{g}$ for $5 \mathrm{~min}$, followed by air drying and fixation with $4 \%$ paraformaldehyde ( $\mathrm{pH}$ 7.4) for $10 \mathrm{~min}$. The smears were then subjected to $\mathrm{Z}-\mathrm{N}$ staining subsequent to permeabilization with $0.3 \%$ Triton X-100 (Fuzhou Maixin Biotechnology Development Co., Ltd., Fuzhou, China) for $30 \mathrm{~min}$. The smears were flooded with carbol fuchsin solution (Shanghai Sangon Biological Engineering Co., Ltd., Shanghai, China) for $20 \mathrm{~min}$, then rinsed with sterile water, decolorized twice with acid-alcohol for $2 \mathrm{~min}$, then rinsed with sterile water, counterstained with methylene blue (Shanghai Sangon Biological Engineering Co. Ltd.) for $2 \mathrm{~min}$, rinsed with sterile water and allowed to air dry. The GeneXpert test was performed to detect TBM according to the reported method (13).

Microscopic observation. The well-stained smears of each CSF specimen were observed successively using an Olympus BX-51 light microscope (Olympus Corporation, Tokyo, Japan) under transmitted light (magnification, x1,000) and under fluorescence with the green-excitation wavelength (546-590 nm; magnification, x1,000). AFB were observed as red, thin and slightly curved structures under transmitted light. Fuchsin-stained AFB were also directly detectable by green-excitation wavelength under a fluorescent microscope, and orange-red emission could be observed without the use of a fluorescent dye, for example, auramine $\mathrm{O}$ or auramine-rhodamine.

Recording and reporting of results. The results were reported according to the Revised National Tuberculosis Control Programme guidelines (14). All smears were read and classified as positive or negative by two types of observational methods. A single acid-fast bacillus was considered as a cutoff point for a positive result. For each smear, 100 fields were examined by two experienced observers prior to declaring a smear negative or positive.

Statistical analysis. SPSS statistical software (version 14.0; SPSS, Inc., Chicago, IL, USA was employed for statistical analysis. Subsequent to reading of the smears under transmitted light and fluorescence, the results were compared through use the two types of observational methods. The $\chi^{2}$ test was used to compare the detection rate of the two methods. $\mathrm{P}<0.05$ was considered to indicate a statistically significant difference.

\section{Results}

Characteristics of patients. Twenty-seven definitive TBM cases with 52 smears were identified, among which $15 \mathrm{CSF}$ samples tested positive by isolation of the organism from culture, 19 CSF samples tested positive through the use of conventional Z-N staining and 22 CSF samples tested positive by GeneXpert. In addition, there were 22 probable TBM cases with 39 smears and 50 possible TBM cases with 76 smears. Among the 99 patients with definite, probable or possible TBM, 68 patients were classified as stage 1, and the remaining 31 patients were classified as stage 2 . In the 56 non-tuberculous control cases, there were 17 cases of meningeal carcinomatosis, 10 cases of purulent meningitis, 6 cases of cryptococcal meningitis, 8 cases of leukemia with central nervous system involvement, 4 cases of neurocysticercosis cases and 11 cases of viral encephalitis.

Micrographs of AFB obtained with two observational methods. The fuchsin-stained AFB revealed bright orange-red fluorescing rods under fluorescence or red, lightly curved rods under transmitted light (Fig. 1). The shapes of the AFB were diverse and appeared thin and slightly curved prior to treatment (Fig. 2A and B), however, they became shorter and thicker following treatment (Fig. 2C and D). AFB were clearly observed within the immune cells, including neutrophils, monocytes and lymphocytes. Within the same microscopic field, a greater number of AFB were observed using fluorescence compared with transmitted light, and AFB that were not visible under transmitted light were detectable using fluorescence (Fig. 3).

Performance of fluorescence microscopy and light microscopy. A total of $52 \mathrm{CSF}$ samples were identified by the gold standard method, smear microscopy. The number of CSF samples that tested positive under transmitted light was 44 , while 50 tested positive under fluorescence. Hence, the sensitivity of fluorescence microscopy was $96.2 \%$, whilst that of light microscopy was $84.6 \%(\mathrm{P}<0.05)$. In the negative control group, 6 samples tested positive by fluorescence and light 
A

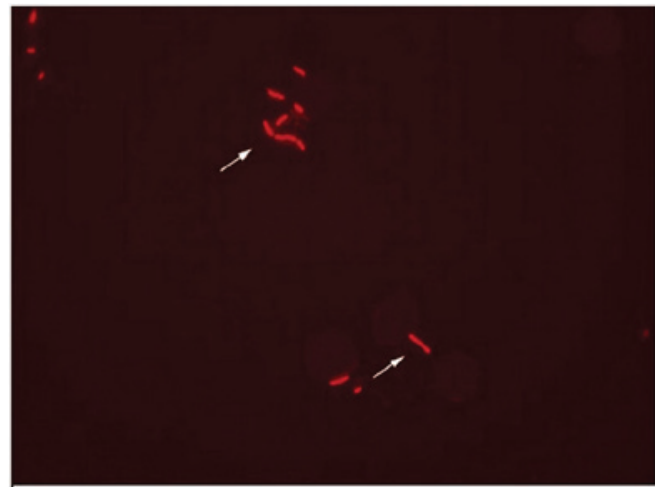

C

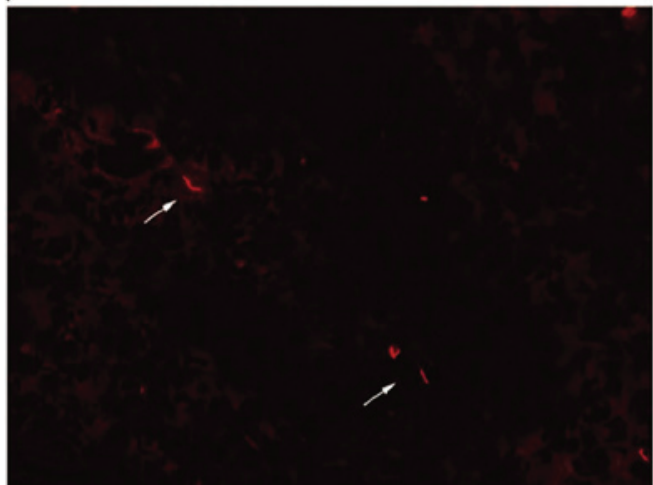

B

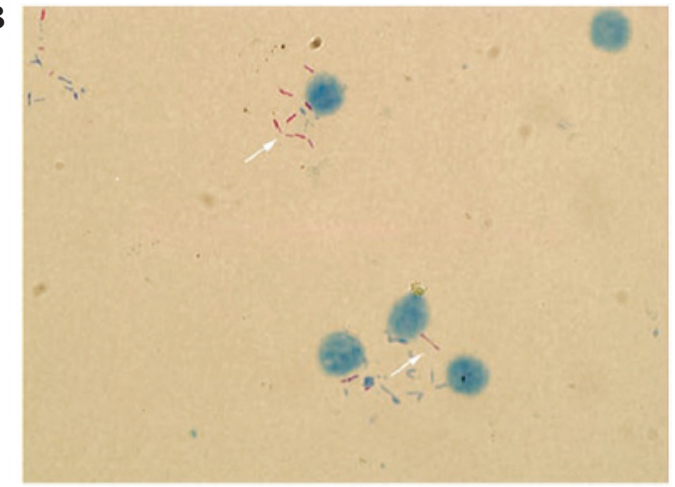

D

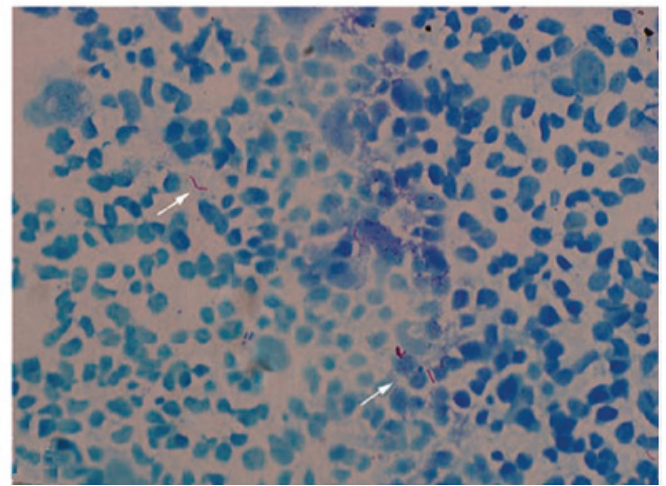

Figure 1. Micrographs of acid-fast bacilli obtained with fluorescence microscopy and transmitted light microscopy (modified Z-N staining). The fuchsin-stained acid-fast bacilli revealed (A) bright orange-red fluorescing rods (shown by arrows) under fluorescence and (B) red, lightly curved rods (shown by arrows) under transmitted light (magnification, x1,000). These rods were also visible (shown by arrows) by (C) fluorescence and (D) transmitted light microscopy at a lower magnification (x800).

A

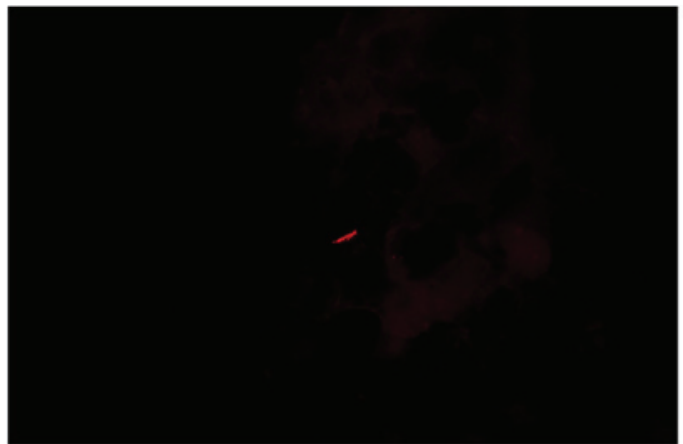

$\mathrm{C}$

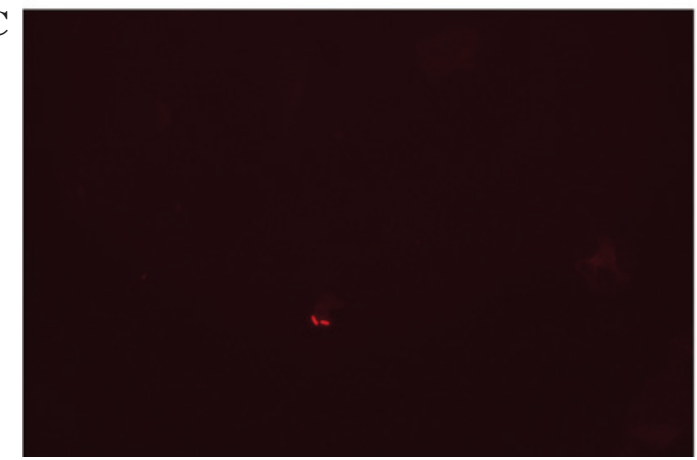

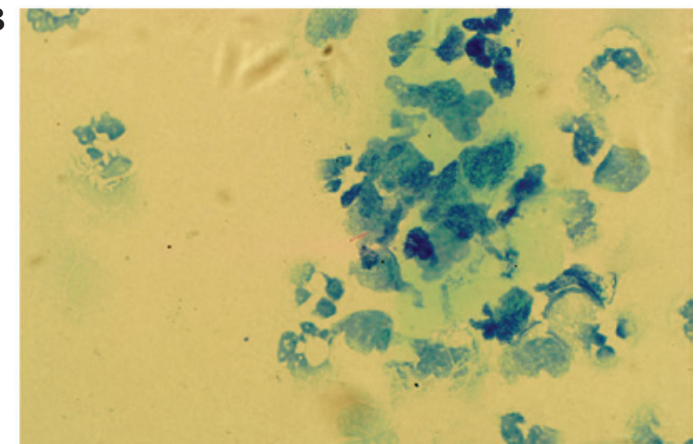

D

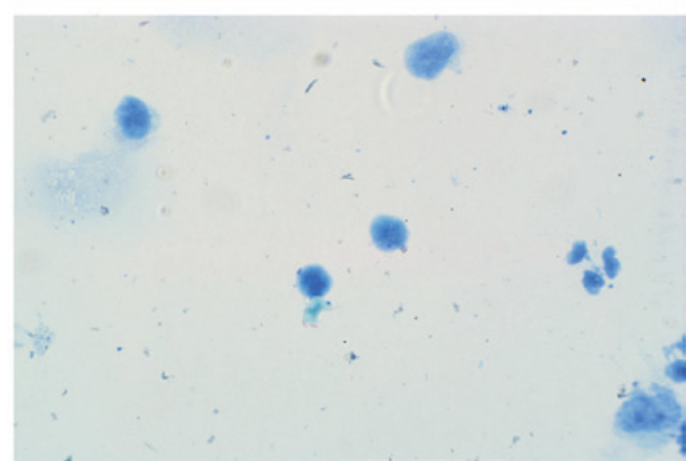

Figure 2. Comparison of the morphologic changes prior to and following treatment (modified Z-N staining; magnification, $\mathrm{x} 1,000)$ ). The shapes of the acid-fast bacilli were diverse and appeared thin and slightly curved prior to treatment, and are shown (A) under fluorescence and (B) under transmitted light. However, the bacilli became shorter and thicker following treatment, as shown (C) under fluorescence and (D) under transmitted light.

microscopy, so the positive predictive value of fluorescence microscopy was $89.3 \%$ and of light microscopy was $88.0 \%$, and the specificities of fluorescence microscopy and light microscopy were $89.3 \%$. The negative predictive value of fluorescence microscopy was $96.2 \%$ and of light microscopy was $86.2 \%$. A comparison of fluorescence microscopy observations 

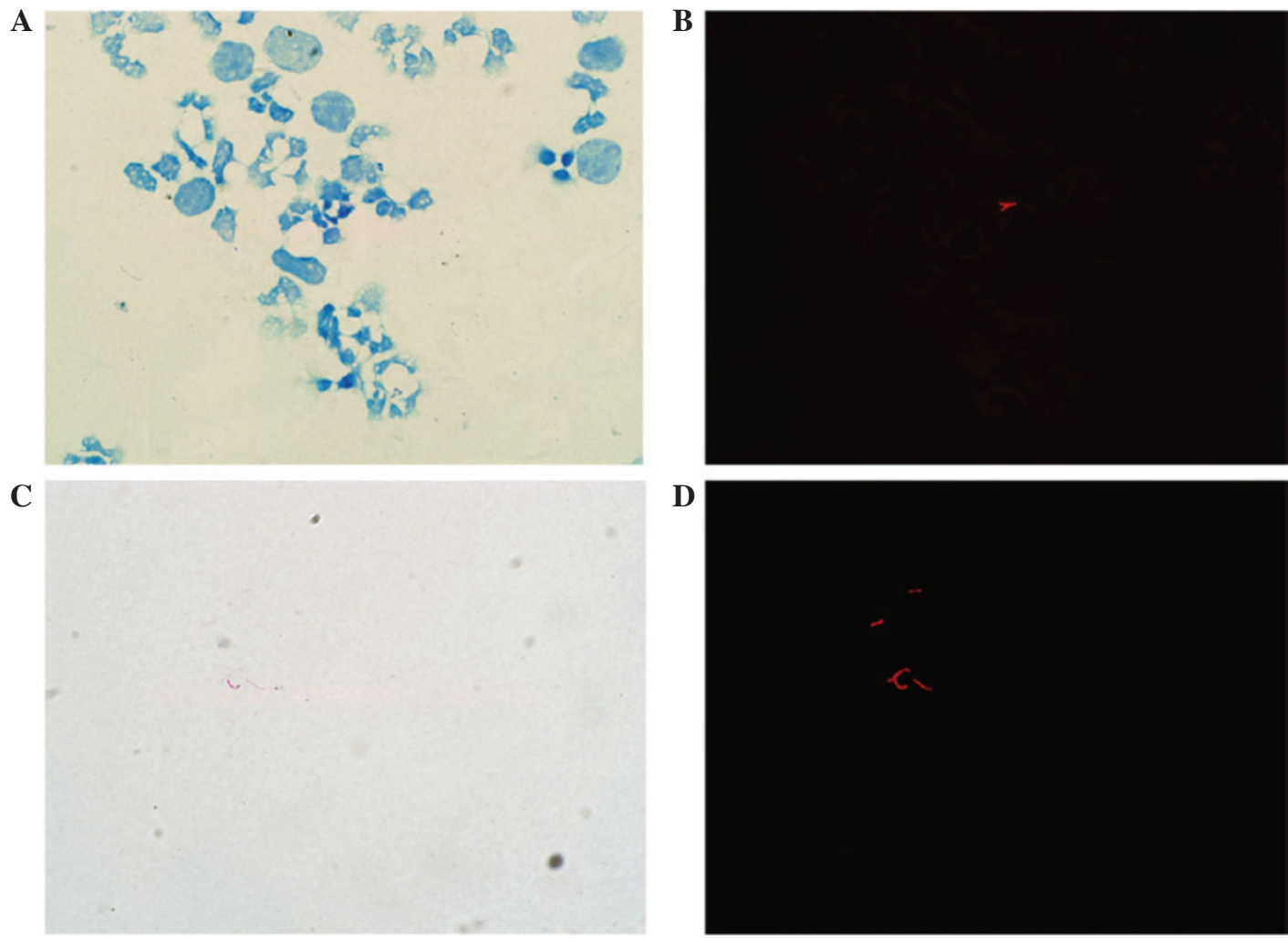

Figure 3. Acid-fast bacilli as observed by light microscopy and fluorescence microscopy in the same microscopic field (modified Z-N staining). At low magnification (x800) acid-fast bacilli were (A) not detectable under transmitted light but (B) were detectable using fluorescence. At higher magnification (x1,000), acid-fast bacilli (C) remained undetectable under transmitted light but (D) were detectable using fluorescence.

and light microscopy observations for the different groups are summarized in Table I. When the results for either patients or samples were analyzed, a statistically significant difference $(\mathrm{P}<0.05)$ was identified in the positive rate of $\mathrm{Z}-\mathrm{N}$ staining between fluorescence microscopy and light microscopy. When a sample analysis was conducted, the positive rate was $57.5 \%$ with light microscopy observation and $73.1 \%$ with fluorescence microscopy observation $(\mathrm{P}<0.05)$. When a patient analysis was conducted, light microscopy observations indicated a positivity of $82.8 \%$, whilst fluorescence microscopy observations revealed a positivity of $90.9 \%(\mathrm{P}<0.05)$.

Comparison of disease duration prior to hospitalization and positive rate between the two observational methods. The positive rate of samples at stage 1 was $86.8 \%(59 / 68)$ by fluorescence microscopy observation, and $95.6 \%(65 / 68)$ by fluorescence microscopy observation. At stage 2, the positive rate of fluorescence microscopy and light microscopy observation was $77.4 \%$ (24/31) and 77.4\% (24/31), respectively (Table II). The positive rate between fluorescence microscopy and light microscopy was significantly different at stage 1 $(\mathrm{P}<0.05)$. There was no statistically significant difference between the two methods for the diagnosis of TBM at stage 2 ( $\mathrm{P}>0.05)$. The positive rate of fluorescence microscopy observation between stages 1 and 2 was also significantly different $(\mathrm{P}<0.05)$.

Comparison of positive rates of fluorescence microscopy prior to and after treatment. Initial lumbar puncture CSF was defined as the CSF prior to treatment, and the CSF of the patients who received antituberculosis drugs and had $<10$ white blood cells/ $\mu 1$ were defined as CSF after treatment. Of the 77 CSF specimens classified into the 'prior to' treatment group, 61 tested positive. Of the 47 post-treatment CSF specimens, 29 tested positive. The positive rate prior to treatment was higher compared with that after treatment $(\mathrm{P}<0.05)$.

\section{Discussion}

The World Health Organization estimated that, in 2010, there were 8.8 million new cases of tuberculosis of all forms globally, and 1.45 million mortalities from the infection (14). Although TBM represents $\sim 1 \%$ of all cases of tuberculosis, $\sim 50 \%$ of those affected will become severely disabled or succumb to mortality as a result of the disease (15). A diagnosis of TBM can be made by various techniques, including clinical, immunological and radiological methods, and the identification of Mycobacterium tuberculosis in samples is the most accurate and reliable method of diagnosis of tuberculosis (16). However, the number of effective diagnostic techniques was limited prior to the investigation of modified Z-N staining from China. Shapiro and Hänscheid reported that M. tuberculosis stained too faintly by fuchsin to be detectable under transmitted light could successfully be detected by green-excited orange-red fluorescence (17). Cryptosporidium parvum and Isospora belli oocysts stained with fuchsin also fluoresce bright red under green light (546 nm) (18). However, this study was not a case-control study, and requires repetition using a larger sample size. Based on this, the present group performed a comparative study to examine the reliability of combined modified Z-N 
Table I. Evaluation of the two observational methods in each diagnostic group.

Positive results, $\mathrm{n}(\%)$

\begin{tabular}{lllcr} 
Analysis type & Group & N & Fluorescence microscopy & Light microscopy \\
\hline Patients & Definite & 27 & $27(100)$ & $27(100)$ \\
& Probable & 22 & $17(77.3)$ & $11(50.0)$ \\
& Possible & 50 & $46(92.0)$ & $44(88.0)$ \\
\multirow{3}{*}{ Sample } & Control & 56 & $6(10.7)$ & $6(10.7)$ \\
& Definite & 52 & $41(78.8)$ & $35(67.3)$ \\
& Probable & 39 & $28(71.8)$ & $16(41.0)$ \\
& Possible & 76 & $53(69.7)$ & $45(59.2)$ \\
& Control & 56 & $6(10.7)$ & $6(10.7)$ \\
\hline
\end{tabular}

Table II. Comparison of positive rates of tuberculous meningitis diagnosis for the two observational methods according to disease duration prior to hospitalization.

\begin{tabular}{lcc}
\hline & \multicolumn{2}{c}{ No. of samples } \\
\cline { 2 - 3 } $\begin{array}{l}\text { Disease duration } \\
\text { and diagnosis }\end{array}$ & $\begin{array}{c}\text { Light } \\
\text { microscopy }\end{array}$ & $\begin{array}{c}\text { Fluorescence } \\
\text { microscopy }\end{array}$ \\
\hline$<1$ month & 59 & 65 \\
Positive & 9 & 3 \\
Negative & & \\
$\geq 1$ month & 24 & 24 \\
Positive & 7 & 7 \\
Negative & & \\
\hline
\end{tabular}

staining and fluorescence microscopy observation for AFB. A good positive rate was achieved with fluorescence microscopy detection of Z-N-stained AFB. The sensitivity of fluorescence microscopy observation was $96.2 \%$ and the specificity was 89.3\%. The sensitivity of fluorescence microscopy observation was $10 \%$ higher than that of light microscopy observation. The results are consistent with the literature (19). We hypothesize that the combination of modified Z-N staining and fluorescence microscopy without auramine $\mathrm{O}$ is superior to using light microscopy alone.

It is generally accepted that fluorescence microscopy has the advantages of increased sensitivity and reduced workload (12,20-26). However, conventional fluorescence microscopic observation of AFB requires the use of dyes such as auramine-rhodamine or acridine, which are relatively expensive and whose fluorescence is rapidly quenched. Furthermore, they are toxic substances and carcinogenic. The modified method presented in the current study involves the use of fuchsin instead of auramine $\mathrm{O}$ and observed the auto-fluorescence of fuchsin-stained AFB through a green excitation filter (546-590 $\mathrm{nm}$ ) under a fluorescence microscope. The fluorescent contrast caused by fuchsin-stained AFB was observed more readily compared with the red of AFB against a blue background in light microscopy. Furthermore, the background of stained slides under fluorescence is brighter than under transmitted light, facilitating the focus on and examination of smears with fewer bacilli for the laboratory technician. The well-stained smears may be stored for an extended duration prior to review. The examination time of fluorescence microscopy observation was $\sim 50 \%$ more rapid compared with light microscopy examination, consolidating upon the results reported by Xia et al (27). The fuchsin-stained AFB exhibited a bright orange-red fluorescence, clustered or diffused distribution and thin and slightly curved bacilli. It was also observed that the AFB were markedly shorter and thicker subsequent to antituberculosis treatment. It is reported that bacteria are able to enter into a cell-less state known as L-form conversion under certain conditions (28). L-forms display a variety of morphological shapes, including bulbiform, rod-shaped bacteria and filamentous structures. The use of isoniazide is a cause of AFB entering into L-form (29).

In the current study, the positive rate of AFB was higher with fluorescence microscopy compared with light microscopy, irrespective of whether the results were analyzed according to the number of samples or patients in each group. The positive rate in the definite group was the highest. There was no statistically significant difference between the probable and possible groups. This suggested that the international TBM diagnostic criteria may assist the optimization of the diagnostic process of TBM. Once analysis of all patients was complete, fluorescence microscopy observation indicated 90.9\% positivity, whereas light microscopy observation indicated $82.8 \%$ positivity. Furthermore, with the same microscopic field, a greater number of AFB were detectable using fluorescence compared with transmitted light, and more AFB that were undetectable under transmitted light could be clearly seen under fluorescence. Equivocal smears encountered with fluorescence microscopy may then be observed under transmitted light directly. Therefore, fluorescence microscopy may help to focus bacilli and light microscopy may then assist in distinguishing bacilli. This is likely to largely avoid false positive results. The combination of the modified Z-N staining and fluorescence microscopy should greatly improve the diagnostic value of CSF smears, particularly those with a low density of bacilli that are likely to be missed by light microscopy observation, and reduce the inconvenience to microscopists. In countries with a high tuberculosis burden, fluorescence microscopy was not previously widely used due to the expense 
involved in purchasing and maintaining fluorescence microscopy materials, which is far greater compared with that of light microscopy. However, an evaluation in Thailand found that the total cost of fluorescence microscopy operated in the National Tuberculosis Reference Laboratory (NTRL) in Bangkok, Thailand, was similar to that of light microscopy performed in the NTRL and in four regional Thai laboratories (30). Thus, fluorescence microscopy and light microscopy should be used in combination to improve the detection rate. The positive rate of AFB was higher when the analysis was conducted on the basis of patient numbers than when analyzed according to sample numbers in the same group, suggesting that the sensitivity will increase if the number of specimens for each patient is increased.

In the present study, the association between disease duration prior to hospitalization and the positive rate of the two observational methods was observed. With the use of fluorescence microscopy observation, a difference was observed between stages 1 and 2, indicating the earlier the inspection, the higher the positive rate. The positive rate was $20 \%$ higher at stage 1 compared with stage 2 . At stage 1,65 patients tested positive with fluorescence microscopy observation while 59 patients were positive with light microscopy observation. Thus, missed diagnosis as a result of numerous false negatives was largely avoided by fluorescence microscopy observation. Furthermore, we hypothesize that a higher proportion of patients will be detected earlier using the modified observational method, compared with previous methods.

The positive rate of fluorescence microscopy was $72.2 \%$ prior to treatment compared with a $61.7 \%$ post-treatment positive rate $(\mathrm{P}<0.05)$. This suggests that $\mathrm{Z}-\mathrm{N}$ staining results may be useful for predicting clinical effects. However, the present study also observed that the post-treatment positive rate of $\mathrm{Z}-\mathrm{N}$ staining remained high. Thus, it may be speculated that the bacteria may be dead but continue to exist in the CSF for a long period of time. Furthermore, in a number of immunocompromised patients, the immune function cannot be activated effectively, so removal of the bacteria is not possible. The result of Z-N staining may remain positive even when there are $<10$ white blood cells $/ \mu 1$ in the CSF; for such patients, an improvement of clinical manifestations was not evident.

Overall, there are a number of advantages of this modified technique. Firstly, the combination of the modified Z-N staining and fluorescence microscopy is convenient and sufficiently sensitive to examine samples for the presence of AFB rapidly, especially for the detection of AFB in CSF. On this basis, a greater number of AFB may be observed in a field using fluorescence rather than transmitted light. The work efficiency of the microscopist should be improved and the workload reduced. Secondly, the microscopist may compare the fluorescence and transmitted light images on the same smears simultaneously, to clarify the AFB status. Finally, $\mathrm{Z}-\mathrm{N}$ dyeing followed by fluorescence microscopy observation is utilized instead of auramine $\mathrm{O}$ or rhodamine, which will reduce the waste in the laboratory and reduce the harm to microscopists. Thus, this method may be easily carried out in the general laboratory.

In the present study, 6 patients in the negative control group tested positive, including 4 patients with meningeal carcinomatosis, 1 patient with neurocysticercosis and 1 patient with viral encephalitis. Each of the 6 patients had a diagnostic score of $>6$ according to the international TBM diagnostic criteria, and one of the patients with meningeal carcinomatosis was classified into the probable group. It remains uncertain as to whether these were true- or false-positives. However, there are reports concerning the coexistence of tuberculosis and cancer $(31,32)$ and the coexistence of tuberculosis and viral encephalitis (33). Initially, for a patient with tuberculosis, a decline in cellular immune function creates favorable conditions for tumorigenesis. Similarly, tumor cells are able to produce immunosuppressive factors that may reduce the body's immune function and increase the chances of tuberculosis (34). Secondly, inflammation associated with infection may contribute to carcinogenesis, and inflammation has powerful effects on tumor development (32). Neutrophils are the initial recruited effectors of the acute inflammatory response. Reactive nitrogen and oxygen species released from inflammatory cells are able to bind to DNA, inducing tumorigenesis and metastasis (32). Thirdly, during tissue repair in patients with pulmonary tuberculosis, there is increased cell proliferation and angiogenesis, and the epithelium is increasingly prone to metaplasia. Furthermore, there may be an association between tuberculosis and cancer or tuberculosis and viral encephalitis. However, whether the 6 patients in the control group who tested positive actually had tuberculosis was not determined. The clinicians did not further examine the patients subsequent to the detection of malignant cells or etiological diagnosis from the CSF.

In conclusion, the combination of modified Z-N staining and fluorescence microscopy without auramine $\mathrm{O}$ is a reliable alternative method for the diagnosis of tuberculous meningitis and has many favorable attributes, and thus may be widely used in countries with a high tuberculosis burden.

\section{Acknowledgements}

The present study was supported by the Medical Science Research of Hebei Province, Shijiazhuang, China (grant no. 20120319).

\section{References}

1. Yu HY, Hu FS, Xiang DR and Sheng JF: Clinical management of tuberculous meningitis: Experiences of 42 cases and literature review. Neurol Sci 35: 303-305, 2014

2. Elkington PT: Tuberculosis: Time for a new perspective? J Infect 66: 299-302, 2013.

3. Zou Y, He J, Guo L, Bu H and Liu Y. Prediction of cerebrospinal fluid parameters for tuberculous meningitis. Diagn Cytopathol 43: 701-704, 2015

4. Marais S, Thwaites G, Schoeman JF, Török ME, Misra UK, Prasad K, Donald PR, Wilkinson RJ and Marais BJ: Tuberculous meningitis: A uniform case definition for use in clinical research. Lancet Infect Dis 10: 803-812, 2010.

5. Thwaites GE: Advances in the diagnosis and treatment of tuberculous meningitis. Curr Opin Neurol 26: 295-300, 2013.

6. Chen P, Shi M, Feng GD, Liu JY, Wang BJ, Shi XD, Ma L, Liu XD, Yang YN, Dai W, et al: A highly efficient Ziehl-Neelsen stain: Identifying de novo intracellular Mycobacterium tuberculosis and improving detection of extracellular M. tuberculosis in cerebrospinal fluid. J Clin Microbiol 50: 1166-1170, 2012.

7. Feng GD, Shi M, Ma L, Chen P, Wang BJ, Zhang M, Chang XL, Su XC, Yang YN, Fan XH, et al: Diagnostic accuracy of intracellular Mycobacterium tuberculosis detection for tuberculous meningitis. Am J Respir Crit Care Med 189: 475-481, 2014. 
8. Ulrichs T, Lefmann M, Reich M, Morawietz L, Roth A, Brinkmann V, Kosmiadi GA, Seiler P, Aichele P, Hahn H, et al: Modified immunohistological staining allows detection of Ziehl-Neelsen-negative Mycobacterium tuberculosis organisms and their precise localization in human tissue. J Pathol 205: 633-640, 2005.

9. Yeager H Jr, Lacy J, Smith LR and LeMaistre CA: Quantitative studies of mycobacterial populations in sputum and saliva. Am Rev Respir Dis 95: 998-1004, 1967.

10. Thwaites GE, Chau TT and Farrar JJ: Improving the bacteriological diagnosis of tuberculous meningitis. J Clin Microbiol 42: 378-379, 2004.

11. Radhakrishnan VV and Mathai A: Correlation between the isolation of Mycobacterium tuberculosis and estimation of mycobacterial antigen in cisternal, ventricular and lumbar cerebrospinal fluids of patients with tuberculous meningitis. Indian J Pathol Microbiol 36: 341-347, 1993.

12. Marais BJ, Brittle W, Painczyk K, Hesseling AC, Beyers N, Wasserman E, van Soolingen D and Warren RM: Use of light-emitting diode fluorescence microscopy to detect acid-fast bacilli in sputum. Clin Infect Dis 47: 203-207, 2008.

13. Nhu NT, Heemskerk D, Thu do DA, Chau TT, Mai NT, Nghia HD, Loc PP, Ha DT, Merson L, Thinh TT, et al: Evaluation of GeneXpert MTB/RIF for diagnosis of tuberculous meningitis. J Clin Microbiol 52: 226-233, 2014.

14. World Health Organization: Global Tuberculosis Control: WHO Report 2010. World Health Organization, Geneva, 2010

15. Thwaites GE, van Toorn R and Schoeman J: Tuberculous meningitis: More questions, still too few answers. Lancet Neurlo 12: 999-1010, 2013.

16. Zakham F, Akrim M, El Mzibri M, Benjouad A, El Aouad R and Ennaji MM: Rapid screening and diagnosis of tuberculosis: A real challenge for the mycobacteriologist. Cell Mol Biol (Noisy-le-grand) 58: (Suppl): S1632-S1640, 2012.

17. Shapiro HM and Hänscheid T: Fuchsin fluorescence in Mycobacterium tuberculosis: The Ziehl-Neelsen stain in a new light. J Microbiol Methods 74: 119-120, 2008.

18. Varea M, Clavel A, Doiz O, Castillo FJ, Rubio MC and Gómez-Lus R: Fuchsin fluorescence and autofluorescence in Cryptosporidium, Isospora and Cyclospora oocysts. Int J Parasitol 28: 1881-1883, 1998.

19. Steingart KR, Henry M, Ng V, Hopewell PC, Ramsay A, Cunningham J, Urbanczik R, Perkins M, Aziz MA and Pai M: Fluorescence versus conventional sputum smear microscopy for tuberculosis: A systematic review. Lancet Infect Dis 6: 570-581, 2006.

20. Kivihya-Ndugga LE, van Cleeff MR, Githui WA, Nganga LW, Kibuga DK, Odhiambo JA and Klatser PR: A comprehensive comparison of Ziehl-Neelsen and fluorescence microscopy for the diagnosis of tuberculosis in a resource-poor urban setting. Int J Tuberc Lung Dis 7: 1163-1171, 2003.
21. Ulukanligil M, Aslan G and Tasci S: A comparative study on the different staining methods and number of specimens for the detection of acid fast bacilli. Mem Inst Oswaldo Cruz 95: 855-858, 2000.

22. Hänscheid T: The future looks bright: Low-cost fluorescent microscopes for detection of Mycobacterium tuberculosis and Coccidiae. Trans R Soc Trop Med Hyg 102: 520-521, 2008.

23. Lehman LG, Ngapmen Yamadji AL, Ngo Sack F and Bilong Bilong CF: The CyScope ${ }^{\circledR}$ fluorescence microscope, a reliable tool for tuberculosis diagnosis in resource-limited settings. Am J Trop Med Hyg 83: 906-908, 2010.

24. Habtamu M, van den Boogaard J, Ndaro A, Buretta R, Irongo CF, Lega DA, Nyombi BM and Kibiki GS: Light-emitting diode with various sputum smear preparation techniques to diagnose tuberculosis. Int J Tuberc Lung Dis 16: 402-407, 2012.

25. Ba F and Rieder HL: A comparison of fluorescence microscopy with the Ziehl-Neelsen technique in the examination of sputum for acid-fast bacilli. Int J Tuberc Lung Dis 3: 1101-1105, 1999.

26. Prasanthi K and Kumari AR: Efficacy of fluorochrome stain in the diagnosis of pulmonary tuberculosis co-infected with HIV. Indian J Med Microbiol 23: 179-181, 2005

27. Xia H, Song YY, Zhao B, Kam KM, O'Brien RJ, Zhang ZY, Sohn H, Wang W and Zhao YL: Multicentre evaluation of Ziehl-Neelsen and light-emitting diode fluorescence microscopy in China. Int J Tuberc Lung Dis 17: 107-112, 2013

28. Willett HP and Thacore H: Formation of spheroplasts of Mycobacterium tuberculosis by lysozyme in combination with certain enzymes of rabbit peritoneal monocytes. Can J Microbiol 13: 481-487, 1967.

29. Ye HP and Xu HP: Research about L-form of Mycobacterium tuberculosis. Jiang Xi Zhong Yi Xue Yuan Xue Bao 12: 92-93, 2000 (In Chinese).

30. Sohn H, Sinthuwattanawibool C, Rienthong S and Varma JK: Fluorescence microscopy is less expensive than Ziehl-Neelsen microscopy in Thailand. Int J Tuberc Lung Dis 13: 266-268, 2009.

31. Silva DR, Valentini Jr DF, Müller AM, de Almeida CP and Dalcin Pde T: Pulmonary tuberculosis and lung cancer: Simultaneous and sequential occurrence. J Bras Pneumol 39: 484-489, 2013.

32. Coussens LM and Werb Z: Inflammation and cancer. Nature 420: 860-867, 2002

33. Aibar-Arregui MA, de Escalante-Yangüela B, Tejero-Juste C and Martín-Fortea MP: Mixed meningoencephalitis caused by Mycobacterium tuberculosis and varicella zoster virus. Rev Neurol 48: 91-93, 2009 (In Spanish).

34. Cicenas $S$ and Vencevicius V: Lung cancer in patients with tuberculosis. World J Surg Oncol 5: 22, 2007. 